\title{
Interaction of two modulational instabilities in a semiconductor resonator
}

\author{
G. Kozyreff and S. J. Chapman \\ Mathematical Institute, 24-29 St. Giles', Oxford OX1 3LB, United Kingdom \\ M. Tlidi \\ Optique Nonlinéaire Théorique, Universite Libre de Bruxelles, Campus Plaine, Case Postale 231, B-1050 Bruxelles, Belgium
}

(Received 4 December 2002; published 15 July 2003)

\begin{abstract}
The interaction of two neighboring modulational instabilities in a coherently driven semiconductor cavity is investigated. First, an asymptotic reduction of the general equations is performed in the limit of a nearly vertical input-output characteristic. Next, a normal form is derived in the limit where the two instabilities are close to one other. An infinity of branches of periodic solutions are found to emerge from the unstable portion of the homogeneous branch. These branches have a nontrivial envelope in the bifurcation diagram that can either smoothly join the two instability points or form an isolated branch of solutions.
\end{abstract}

DOI: 10.1103/PhysRevE.68.015201

PACS number(s): 05.45.-a, 42.65.Sf

Transverse patterns in nonlinear optical resonators have been the subject of intense research since the pioneering work by Moloney and Gibbs and Lugiato and Lefever [1]. Semiconductor cavities, in particular, have received special attention owing to their associated mature technology. Recently, hexagonal structures [2], bright cavity solitons [3,4], and dark cavity solitons [4] were experimentally observed in these devices and a reasonable agreement was found with the model equations put forward in Ref. [5]. On general grounds, it is understood that such spatial structures, whether localized or extended, result from modulational instabilities (MI) [6-8]. Moreover, as shown numerically in Ref. [9], the localized solutions and the spatially periodic ones that bifurcate from the MI's are intimately related. This demonstrates the importance of MI's when studying the complex spatial dynamics of these systems.

Previous work revealed that more than one MI can coexist, each associated with different critical wave numbers [10]. The purpose of the present paper is to provide an analytical understanding of how two such MI can interact in this system. This question has obviously a much broader scope than semiconductor cavities. Previous studies have shown that coexisting pattern-forming instabilities can give rise in two dimensions to quasicrystals [11] or superlattices [12] through resonance between unstable modes in nonequilibrium spatially extended systems. However, to the best of our knowledge, the kind of interaction reported here has not been addressed so far.

Due to the complicated microscopic structure of the material and because of the diffusion of carriers, only numeric [9] or semianalytic [13] theoretical results are available for these devices. Even the characteristic polynomial has to be studied numerically [10]. As a preliminary step in our investigation, therefore, we will derive a simpler, more tractable equation that retains the relevant spatial dynamics of the model in Ref. [5].

We thus start from the model in Ref. [5], although we normalize the variables with respect to the lasing threshold values (in the absence of injected field) in order to reduce the number of free parameters. If we consider a bulk semiconductor cavity driven by a constant electric field amplitude $Y$ at frequency $\omega_{i}$, the equations for the dimensionless field $F$ and carrier (real) variable $Z$ are

$$
\begin{gathered}
\frac{\partial F}{\partial t}=i \theta F+(1+i \alpha) Z F-i \nabla^{2} F+Y, \\
\frac{\partial Z}{\partial t}=\gamma\left[P-Z-(1+2 Z)|F|^{2}+D \nabla^{2} Z\right],
\end{gathered}
$$

where time is rescaled to the photon lifetime $\kappa^{-1}, \theta=\left(\omega_{c}\right.$ $\left.-\omega_{i}\right) / \kappa$ is the normalized cavity detuning, $\alpha$ is the linewidth enhancement factor, and $\nabla^{2}$ is the transverse Laplacian. The unit length is the diffraction length $v / \sqrt{2 \kappa \omega_{i}}, v$ being the speed of light. $\gamma=1 /\left(\kappa T_{n}\right)$ is the ratio of the nonradiative carrier recombination time to the photon lifetime and $D$ $=\left(2 \kappa \omega_{i} T_{n} / v^{2}\right) D_{n}$ is the rescaled carrier diffusion constant. Finally, $P$ is the pump parameter; it is related to the injected current $I$ through $P=\left(g N_{t h} / 2 \kappa\right)\left(I / I_{t h}-1\right)$, where $g$ is the differential gain and $N_{t h}, I_{t h}$ are the lasing threshold values of the electron density and the electric current, respectively. They are themselves related to the transparency density $N_{0}$ and the volume of the active region $V$ through $N_{t h}=N_{0}$ $+\kappa / g, I_{t h}=e V N_{t h} / T_{n}$. In what follows, we only consider pumping currents below the lasing threshold, so that $-g N_{t h} / 2 \kappa<P<0$. For the sake of simplicity, we have neglected the radiative recombination of carriers and, without loss of generality, $Y$ is real. Finally, we note that if the multiple quantum well structure is used, light and matter can interact through a well resolved excitonic line and the corresponding equations are equivalent to Eqs. (1) and (2). The excitonic detuning is $\delta=\left(\omega_{e}-\omega_{i}\right) / \gamma_{e}$, where $\omega_{e}$ is the excitonic resonance and $\gamma_{e}$ is the linewidth; $\delta$ plays essentially the same role as $\alpha$.

For two-level atoms, an analytical treatment based on the smallness of the detunings $\theta$ and $\delta$ leads to a SwiftHohenberg equation (SHE) $[14,15]$. In the present situation, $\delta$ is replaced by $\alpha$, which is generally of order the of 1 , so that the same scheme is not applicable. However, we can simplify Eqs. (1) and (2) while retaining the relevant physics if we can identify the conditions where (i) the bistability is 
nascent, (ii) the modulational instabilities that are responsible for the appearance of spatially structured solutions are in a close vicinity of the switching region, and (iii) the associated unstable wave numbers $k$ are small. Moreover, the problem can be explicitly handled if the cavity detuning $\theta$ vanishes. We therefore begin our study with $\theta=0$. Otherwise, the conditions just stated are implicit and the development that we will present involve much heavier algebraic expressions.

For the homogeneous steady state, one has $Y$ $=Y(P,|F|)$. Nascent bistability corresponds to $\partial Y / \partial|F|$ $=\partial^{2} Y / \partial|F|^{2}=0$. This occurs for the critical values $P_{c}$ $=-9 / 2, \quad F_{c}=(1-i \alpha) \sqrt{3 / 2\left(1+\alpha^{2}\right)}, \quad Z_{c}=-3 / 2, \quad$ and $Y_{c}$ $=\sqrt{27\left(1+\alpha^{2}\right) / 8}$. At this operation point, the steady state is unstable with respect to homogeneous fluctuations. On the other hand, we find from the linear stability analysis that there is a long wavelength instability at this point if

$$
D \simeq D_{c}=8 \alpha / 3\left(1+\alpha^{2}\right) .
$$

With this piece of information, we are in a position to reduce the laser equations to a single scalar partial differential equation by perturbation. We closely follow the procedure exposed in Ref. [15] for two-level atoms. To formalize the requirement of a small hysteresis domain, we introduce a small parameter $\epsilon$ by

$$
P=P_{c}+3 \epsilon^{2} p .
$$

Second, $Y$ is parametrized as $Y=Y_{c}\left(1-\epsilon^{2} p / 2+\epsilon^{3} y / 4\right)$, where the second term anticipates a solvability condition at $O\left(\epsilon^{2}\right)$ of the development. Next, from Eq. (3), we write $D$ $=D_{c}(1+\epsilon d)$. It is also necessary to rescale time and space as $\tau \propto \epsilon^{2} t$ and $\boldsymbol{\xi} \propto \epsilon^{1 / 2} \mathbf{x}$. The most suitable scaling in that respect appears to be $\tau=\epsilon^{2} t /\left[1 / \gamma+D_{c} / \alpha\right]$ and $\xi$ $=\epsilon^{1 / 2} \mathbf{x} / \sqrt{D_{c}}$. Let us then substitute in Eqs. (1) and (2) the following power series for $F$ and $Z$ :

$$
\begin{aligned}
& F(\mathbf{x}, t)=F_{c}[1+\epsilon f(\boldsymbol{\xi}, \tau)+\cdots], \\
& Z(\mathbf{x}, t)=Z_{c}[1+\epsilon z(\boldsymbol{\xi}, \tau)+\cdots] .
\end{aligned}
$$

Collecting like powers of $\epsilon$, we find at dominant order that $z=-f$. It is only at third order in $\epsilon$ through a solvability condition that $f$ is determined. We find [16]

$$
\frac{\partial f}{\partial \tau}=y-f\left(p+f^{2}\right)+(d-f / 2) \nabla^{2} f-a \nabla^{4} f-\nabla^{2} f^{2},
$$

where $\nabla^{2}$ means now $\partial^{2} / \partial \xi_{1}^{2}+\partial^{2} / \partial \xi_{2}^{2}$ and $a \equiv(1$ $\left.-\alpha^{2}\right) /\left(4 \alpha^{2}\right)$. Let us emphasize that the same can be done for nonzero cavity detuning $\theta$, at the cost of increased algebraic complexity. The reduced equation then retains the same form, with the deviation parameters $y, p$, and $d$ preceded by some $\theta$-dependent coefficients. Equation (6) resembles the SHE, which regularly shows up in nonlinear optics $[14,17]$. A notable difference, though, is the occurrence of nonlinear diffusive terms, which are directly imputable to the diffusion of charge carriers in the semiconductor material. These quadratic nonlinearities break the $f \rightarrow-f$ symmetry that exists in the SHE. Other variations of the SHE with nonlinear diffusive terms have been derived for optical parametric oscillators [18] and thermal convection [19], but they preserve this inversion symmetry. The coefficient $a$ in Eq. (6) must be positive, which seems to suggest that Eq. (6) is valid only for $\alpha<1$. In fact, for nonzero $\theta, a$ can be positive for arbitrarily large $\alpha$. To be more concrete, let us consider some typical physical parameters values: $D_{n}=30 \mathrm{~cm}^{2} \mathrm{~s}^{-1}, T_{n}=10^{-9} \mathrm{~s}$, $\kappa=5 \times 10^{11} \mathrm{~s}^{-1}, \omega_{i}=2.2 \times 10^{15} \mathrm{~s}^{-1}, \alpha=3$. The corresponding reduced diffusion parameter $D$ equals 0.67 , and it is close to $D_{c}(\theta)$ provided that $\theta \simeq 1.66$. For this value of $\theta$ and $\alpha=3$, we find that $a=0.58$. In order to check the validity of the above developments, we have compared bifurcation diagrams obtained from Eqs. (1) and (2) and from Eq. (6) and found quantitative agreement with $\epsilon=0.05$ in Eq. (4) although, for some parameter values, $\epsilon$ had to be much smaller [16].

We are now in a good position to study the interaction of MI's. By contrast with the system (1) and (2), the linear stability analysis of the homogeneous steady state can be done explicitly and effortlessly with the modified SHE. The homogeneous steady state is given implicitly by $y=f_{s}(p$ $\left.+f_{s}^{2}\right)$. Introducing perturbations of the form $\exp (\lambda \tau+i \mathbf{k} \cdot \boldsymbol{\xi})$ around $f_{s}$ in Eq. (6) yields the dispersion relation

$$
\lambda=-p-3 f_{s}^{2}-\left(d-5 f_{s} / 2\right) k^{2}-a k^{4} .
$$

Equating simultaneously $\lambda$ and $\partial \lambda / \partial k$ to zero, the thresholds for modulational instability are found to be

$$
f_{s}=f_{ \pm}=\frac{10 d \pm 4 \sqrt{a\left[12 d^{2}+(25-48 a) p\right]}}{25-48 a},
$$

with critical wave numbers $k_{ \pm}=\sqrt{\left(5 f_{ \pm}-2 d\right) / 4 a}$, provided that $5 f_{ \pm}>2 d$. Two possible situations where both thresholds simultaneously exist are depicted schematically in Fig. 1. The fact that $k_{-} \neq k_{+}$and that $f_{-} \neq-f_{+}$results from the nonlinear diffusive terms in Eq. (6) and could not happen in the usual, symmetric, SHE.

Inspection of Eq. (8) reveals that, as $p$ approaches $p^{*}$ $=12 d^{2} /(48 a-25)$, the two instability points $f_{ \pm}$tend to a common value $f^{*}=-5 p^{*} / 6 d$, with wave number $k^{*}$ $=\sqrt{12 f^{*} / 5}$. In the vicinity of this point, we can describe the interaction between the two instabilities analytically. In one dimension, we introduce a new expansion parameter $\bar{\epsilon}$ and two bifurcation parameters $\Delta p$ and $\Delta y$ such that $p=p^{*}$ $+3 \bar{\epsilon}^{2} \Delta p$ and $y=f^{*}\left(p^{*}+f^{* 2}\right)+\bar{\epsilon}\left(p^{*}+3 f^{* 2}\right) \Delta y$. New slow time and space are introduced as $\sigma=3 \bar{\epsilon}^{2} \tau$ and $\eta$ $=6 \bar{\epsilon} \xi / 5 k^{*}$. Then, by a weakly nonlinear analysis [16], we find that

$$
f=f^{*}+\bar{\epsilon} \Delta y+\bar{\epsilon}\left[A(\eta, \sigma) \exp \left(i k^{*} \xi\right)+\text { c.c. }\right]+O\left(\bar{\epsilon}^{2}\right),
$$

where the amplitude $A$ satisfies

$$
\frac{\partial A}{\partial \sigma}=A\left(-\Delta p-\Delta y^{2}-l|A|^{2}\right)-2 i \Delta y \frac{\partial A}{\partial \eta}+\frac{48 a}{25} \frac{\partial^{2} A}{\partial \eta^{2}},
$$



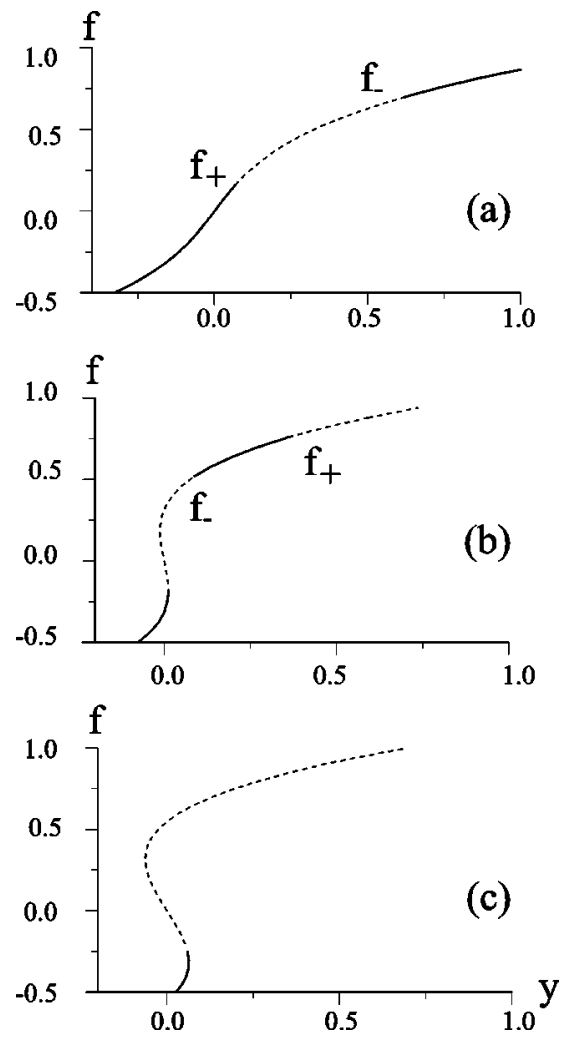

FIG. 1. Three possible outcomes of the linear stability analysis of the homogeneous state of Eq. (6). Full line, stable; dotted line, unstable. The case $p=p^{*}$ corresponds to $f_{+}=f_{-}$in (a) and (b), leading either to a completely stable homogeneous branch or to (c).

with

$$
l=1-13 /(54 a) .
$$

This Ginzburg-Landau equation differs from the one usually encountered in the vicinity of a MI point. Note, in particular, the presence of a complex first-order partial derivative and the way the bifurcation parameters $\Delta p$ and $\Delta y$ appear on the right hand side. It admits an infinity of branches of spatially periodic solutions of the form $A=\rho \exp (i \Delta k \eta)$, where $\rho$ is the oscillation amplitude and $\Delta k$ is a correction to the critical wave number $k^{*}$. Substituting this ansatz in Eq. (10), we obtain $\rho$ as a function of $\Delta y$ and $\Delta k$ through

$$
l \rho^{2}+(\Delta y-\Delta k)^{2}=-\Delta p+\frac{m}{m-1}(\Delta k)^{2},
$$

where $m=1-25 /(48 a)$. To each value of $\Delta k$ corresponds a branch of solution and we thus have a family of curves in the bifurcation diagram, as shown in Fig. 2. A crucial observation is that the envelope of these branches is itself a branch of solutions given by

$$
l \rho_{e n v}^{2}+m \Delta y^{2}=-\Delta p, \quad \Delta k=25 \Delta y /(48 a) .
$$

Let us examine how this "envelope" branch evolves in the bifurcation diagram. Here, we only consider the case $l$ $>0$, for which one can show that this branch is stable [16]. If $m>0$ and $\Delta p<0$, it describes an ellipse that connects the
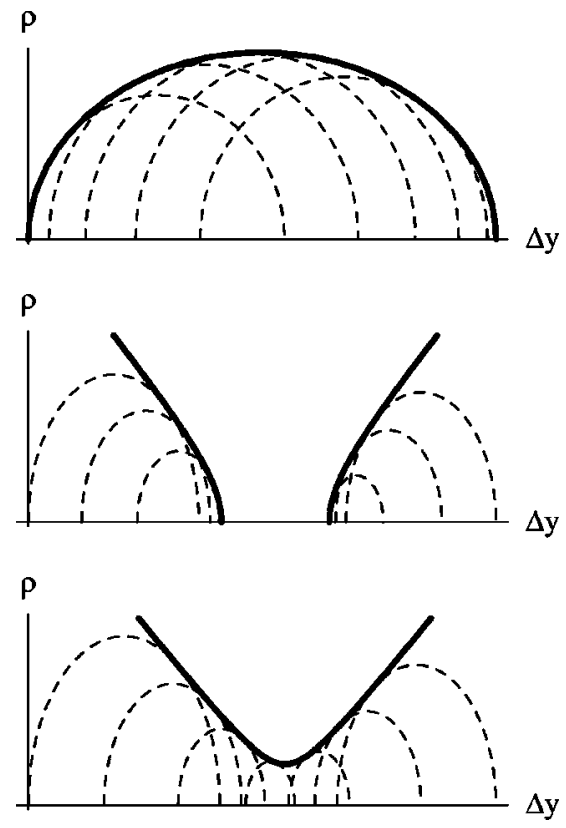

FIG. 2. Typical bifurcation diagrams obtained from Eq. (10) with $l>0$. From top to bottom: $\Delta p<0<m ; m<0<\Delta p ; m, \Delta p$ $<0$. Dotted curves: branches of periodic solutions with fixed wave number $\Delta k$. Full line: "envelope" branch of periodic solution.

two instability points. This connetion is made possible by the fact that the wave number varies along that particular branch. On the other hand, if $m<0$ and $\Delta p>0$, there are two envelope branches which grow in opposite directions. Finally, from this situation, changing the sign of $\Delta p$ leads the two instability points to merge and annihilate in such a way that the envelope solution is nowhere connected to the homogeneous steady state [see Fig. 2]. This proves the existence of isolated branches of solutions in the semiconductor cavity. In this situation, no threshold is associated with the instability. These analytical predictions are well reproduced by the numerical integration of Eq. (6) [see Fig. 3]. Periodic boundary conditions along the transverse direction have been used and the initial condition was taken to be the upper unstable homogeneous steady state plus a small white noise to seed the instability.

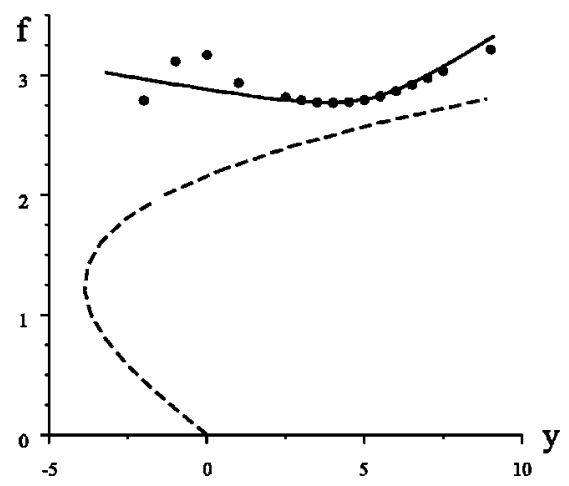

FIG. 3. Isolated branch of spatially periodic solution for $p=$ $-4.67, d=1.5, a=0.4$ in one dimension. Broken line: unstable homogeneous state. Full line: analytical peak value of periodic solutions. Dots: numerical integration of Eq. (6). 
We note that in bifurcation diagrams computed by other authors, e.g. Ref. [3], the periodic branch of solution sometimes presents its concavity upwards, very similarly to Fig. 3 . On the other hand, hexagonal patterns do appear experimentally without threshold [2]. This fact was attributed to device imperfections, which appears perfectly plausible. Here, we worked in the limit of nascent bistability and, in this limit, the isolated branch of periodic solution is found at high field intensities. Therefore, the present analysis cannot directly be applied to the experiment in Ref. [2]. Nevertheless, it shows that thresholdless appearance of periodic patterns can have a dynamical origin, in addition to device imperfections. We note in addition that "collisions" of MI's can, in principle, appear outside the nascent bistability regime and that the reasoning presented here can be done in two dimension as well. Numerical simulations carried out in two dimensions confirm the existence of isolated branch of hexagon solutions.

In conclusion, we have simplified model (1) and (2) into Eq. (6) in the nascent bistability limit. Next, we have derived the normal form (10) for two interacting modulational instabilities with different critical wave numbers. From the un- stable portion of the homogeneous solution emerges a continuous family of periodic branches, parametrized by the wave number. This family of curves assumes a nontrivial envelope in the bifurcation diagram. It is this envelope that should be considered when determining the transverse spatial dynamics of the semiconductor cavity. This envelope can smoothly join the two instability points or form an isolated branch of solutions, in a way that could not be anticipated by only considering a single branch with fixed wave number. After this paper was submitted, Maggipinto et al. published a numerical study of the dynamical system (1) and (2) which completes and supports much of the above analysis [20]. As an extension of this research, it would be interesting to investigate if and how envelope branches influence the bifurcation scenarios leading to quasycristals and superlattices in other spatially extended nonequilibrium systems.

It is a pleasure to thank J. R. Ockendon for fruitful and stimulating discussions. M.T. received support from the Fonds National de la Recherche Scientifique (Belgium). This work was partially supported by the Interuniversity Attraction Pole program of the Belgian government.
[1] J.V. Moloney and H.M. Gibbs, Phys. Rev. Lett. 48, 1607 (1982); L.A. Lugiato and R. Lefever, ibid. 58, 2209 (1987).

[2] V.B. Taranenko, I. Ganne, R.J. Kuszelewicz, and C.O. Weiss, Phys. Rev. A 61, 063818 (2000); R. Kuszelewicz, I. Ganne, I. Sagnes, G. Slekys, and M. Brambilla, Phys. Rev. Lett. 84, 6006 (2000).

[3] S. Barland et al., Nature (London) 419, 699 (2002).

[4] V.B. Taranenko, I. Ganne, R.J. Kuszelewicz, and C.O. Weiss, Appl. Phys. B: Lasers Opt. B72, 377 (2001); V.B. Taranenko, C.O. Weiss, and B. Schapers, Phys. Rev. A 65, 013812 (2001).

[5] M. Brambilla, L.A. Lugiato, F. Prati, L. Spinelli, and W.J. Firth, Phys. Rev. Lett. 79, 2042 (1997).

[6] M. Tlidi, P. Mandel, and R. Lefever, Phys. Rev. Lett. 73, 640 (1994).

[7] N.N. Rosanov, Prog. Opt. 35, 1 (1996).

[8] W.J. Firth and A.J. Scroggie, Phys. Rev. Lett. 76, 1623 (1996).

[9] T. Maggipinto, M. Brambilla, G.K. Harkness, and W.J. Firth, Phys. Rev. E 62, 8726 (2000).

[10] L. Spinelli, G. Tissoni, M. Brambilla, F. Prati, and L.A. Lugiato, Phys. Rev. A 58, 2542 (1998); G. Tissoni, L. Spinelli, M. Brambilla, T. Maggipinto, I.M. Perrini, and L.A. Lugiato, J. Opt. Soc. Am. B 16, 2083 (1999); 16, 2095 (1999); L. Spinelli, G. Tissoni, M. Tarenghi, and M. Brambilla, Eur. Phys. J. D 15, 257 (2001).
[11] Z.H. Musslimani and L.M. Pismen, Phys. Rev. E 62, 389 (2000).

[12] M. Bachir, S. Métens, P. Borckmans, and G. Dewel, Europhys. Lett. 54, 612 (2000).

[13] D. Michaelis, U. Peschel, and F. Lederer, Phys. Rev. A 56, R3366 (1997).

[14] P. Mandel, M. Georgiou, and T. Erneux, Phys. Rev. A 47, 4277 (1993).

[15] P. Mandel, Theoretical Problems in Cavity Nonlinear Optics (Cambridge University Press, Cambridge, 1997), p. 167.

[16] G. Kozyreff and M. Tlidi (unpublished).

[17] J. Lega, J.V. Moloney, and A.C. Newell, Phys. Rev. Lett. 73, 2978 (1994); S. Longhi and A. Geraci, Phys. Rev. A 54, 4581 (1996); A. Barsella, C. Lepers, M. Taki, and P. Glorieux, J. Opt. B: Quantum Semiclassical Opt. 1, 64 (1999).

[18] G.J. de Valcarcel, K. Staliunas, E. Roldan, and V.J. SanchezMorcillo, Phys. Rev. A 54, 1609 (1996); V.J. SanchezMorcillo, E. Roldan, G.J. de Valcarcel, and K. Staliunas, Phys. Rev. E 57, R4911 (1998); V.J. Sanchez-Morcillo and G.J. de Valcarcel, Phys. Lett. A 246, 293 (1998).

[19] H.S. Greenside and M.C. Cross, Phys. Rev. A 31, 2492 (1985).

[20] T. Maggipinto, M. Brambilla, and W.J. Firth, IEEE J. Quantum Electron. 39, 206 (2003). 